\section{Adaptação transcultural do instrumento Tilburg Frailty Indicator (TFI) para a população brasileira}

\author{
Cross-cultural adaptation of the Tilburg Frailty \\ Indicator (TFI) for use in the Brazilian population
}

\begin{abstract}
The current study aimed to adapt the Tilburg Frailty Indicator (TFI), used to assess frailty in the elderly, to the Brazilian population. Conceptual, item, and semantic equivalences were analyzed and the summary version was pre-tested. In the evaluation of conceptual equivalence, the construct for frailty adopted in Brazil demonstrated the same conceptualization as in other cultures where this condition has been investigated. All items included in the original version also showed similarity in the two cultures. High semantic equivalence was observed in the analysis of the items' referential and general meanings. The pre-test showed a high percentage of understanding of items and good acceptance of items by elderly individuals. The results suggest that the TFI version adapted to the Brazilian culture can be a useful tool for assessing health conditions in older Brazilians.
\end{abstract}

Evaluation; Frailty; Aged

\author{
Lívia Maria Santiago 1,2 \\ Laércio Lima Luz 1 \\ Inês Echenique Mattos 1 \\ Robbert J. J. Gobbens 3
}

\section{Introdução}

O termo fragilidade vem sendo utilizado para designar uma condição resultante da desregulação dos sistemas orgânicos e da diminuição das reservas fisiológicas, que se caracteriza pelo aumento da vulnerabilidade e limitação da capacidade de manutenção da homeostase no indivíduo idoso ${ }^{1}$. A fragilidade confere alto risco de desfechos adversos de saúde como quedas, fraturas, hospitalizações e morte ${ }^{2,3}$.

Diferentes instrumentos para avaliação da fragilidade têm sido propostos, com base em duas abordagens distintas. A primeira parte do pressuposto que a fragilidade pode ser mensurada somente com variáveis relativas à condição física; enquanto, a segunda, cada vez mais adotada, inclui variáveis relacionadas aos domínios psicológico e social 1,4,5.

Buscando identificar o instrumento mais adequado para avaliação da fragilidade em idosos brasileiros, realizou-se uma revisão sistemática da literatura nas bases de dados MEDLINE, LILACS e SciELO, sendo identificadas várias propostas 2,3,5,6,7. O Tilburg Frailty Indicator (TFI) foi considerado o instrumento mais adequado ao constructo atual da fragilidade, visto que os outros apresentavam incoerências, tais como basear-se apenas no domínio físico ou incluir desfechos dessa condição 2,6. Em estudo realizado na Holanda, o instrumento demonstrou boas 
propriedades psicométricas 7, porém seu uso está limitado a esse país, sendo necessário avaliar sua adequação para a população brasileira.

Este estudo descreve o processo de adaptação transcultural do TFI para a língua portuguesa, por meio da abordagem "universalista" 8,9.

\section{Métodos}

O TFI é constituído por duas partes, A e B. A parte A é voltada para os determinantes da fragilidade; enquanto, a B refere-se à identificação da fragilidade propriamente dita, sendo foco deste estudo. Essa parte é constituída por 15 questões objetivas, autorreferidas, distribuídas em três domínios: físico, psicológico e social. A maioria das questões é respondida com sim ou não, excetuando-se quatro questões que incluem a opção às vezes. O resultado final é um escore que varia de 0 a 15 pontos. Maior pontuação significa maior nível de fragilidade, ou, alternativamente, escores $\geq$ 5 pontos indicam que o indivíduo é frágil 5 .

Durante o processo de revisão de literatura, foram levantadas informações que possibilitaram a exploração do constructo fragilidade tanto na cultura original quanto na da populaçãoalvo.

Um grupo de especialistas, formado por quatro profissionais das áreas clínica e de pesquisa, discutiu e avaliou a composição do instrumento original quanto à pertinência e relevância de cada um dos seus itens para a avaliação da fragilidade no contexto brasileiro.

A tradução do instrumento original para a língua portuguesa foi efetuada de modo independente por dois profissionais médicos, brasileiros, ambos proficientes no idioma inglês. Procedeu-se à retradução das versões por outros dois tradutores independentes, ambos com amplo domínio do idioma e conhecimento do vocabulário da área de saúde.

Na sequência, uma geriatra com atuação na área de saúde pública e proficiente em inglês realizou a avaliação formal das duas retraduções, comparando-as individualmente ao instrumento original, utilizando dois formulários específicos ${ }^{9}$. Para análise do significado referencial, o avaliador atribuiu escores de correspondência para cada questão, variando de 0 a 100\%; para análise do significado geral, qualificou as questões em uma de quatro categorias: inalterado, pouco alterado, muito alterado ou completamente alterado.

O grupo de especialistas que participou da análise de equivalência conceitual e de itens e o avaliador das retraduções reuniram-se para debater opiniões divergentes quanto à avaliação da equivalência semântica. O grupo discutiu modi- ficações na estrutura de algumas das frases em português, com o objetivo de simplificar enunciados que pudessem gerar dúvidas para os idosos. Realizadas todas as adequações, elaborou-se uma versão-síntese para realização de pré-teste.

Para aplicação da versão-síntese em indivíduos de 60 anos ou mais, respeitou-se a forma utilizada para o instrumento original, com entrevistas face a face. Foi solicitado que os idosos parafraseassem cada questão para avaliação da compreensão das mesmas. Estabeleceu-se, previamente, que as questões que tivessem percentual de entendimento abaixo de $90 \%$ seriam revistas.

Após a análise do pré-teste, o grupo de especialistas reuniu-se para efetuar a revisão do instrumento traduzido. O pesquisador principal do grupo que elaborou o TFI participou de todas as etapas metodológicas dessa adaptação, e a versão final foi submetida à sua apreciação.

\section{Resultados}

Pôde-se constatar que, no Brasil, o constructo fragilidade tem a mesma concepção das demais culturas onde essa condição foi investigada 1,10 . Os 15 itens incluídos no instrumento original apresentam similaridade de conteúdo nas duas culturas 10 .

Na Tabela 1, encontram-se os resultados da análise de equivalência. Quanto ao significado referencial, observou-se elevada similaridade entre as duas retraduções e o instrumento original, variando entre 80 a $100 \%$ para a retradução 1 e entre 90 a $100 \%$ para a 2. Em relação ao significado geral, 5 das 15 questões receberam classificação "pouco alterado" na retradução 1, e somente três, na retradução 2 .

As traduções escolhidas, as alterações realizadas pelo grupo de especialistas e a versão final elaborada encontram-se na Tabela 2. Optou-se por oito questões da tradução 2 e duas da tradução 1. Após as discussões, decidiu-se alterar 13 questões, no sentido de torná-las mais claras e objetivas para melhor compreensão do públicoalvo.

No item 1, omitiu-se a palavra "fisicamente", por se considerar que estava implícita na questão. No item 2, "desejar" foi substituído por "querer", mais utilizado na linguagem popular brasileira.

Para os itens 3 a 8, optou-se pela modificação do enunciado comum para torná-lo menos complexo, e foi efetuada uma adequação necessária ao novo enunciado.

Para os itens 5 e 6, foram substituídas as expressões "deficiência da audição" e "deficiência da visão" pelos seus similares "audição ruim" e 
Avaliação da equivalência semântica entre o instrumento Tilburg Frailty Indicator (TFI) original e versões em português.

\begin{tabular}{|c|c|c|c|c|c|c|c|c|c|}
\hline Item & Original & 1ạ traduçãa & 1ạ retradução & $\%$ * & $\begin{array}{l}\text { Avaliação do } \\
\text { significado } \\
\text { geral ** }\end{array}$ & 2a tradução & $\begin{array}{c}\text { 2a re- } \\
\text { tradução }\end{array}$ & $\%$ * & $\begin{array}{c}\text { Avaliação do } \\
\text { significado } \\
\text { geral ** }\end{array}$ \\
\hline 1 & $\begin{array}{c}\text { Do you feel } \\
\text { physically } \\
\text { healthy? }\end{array}$ & $\begin{array}{c}\text { Você se sente } \\
\text { fisicamente sau- } \\
\text { dável? }\end{array}$ & $\begin{array}{c}\text { Do you feel } \\
\text { physically } \\
\text { healthy? }\end{array}$ & 100 & IN & $\begin{array}{l}\text { Você se sente } \\
\text { fisicamente } \\
\text { saudável? }\end{array}$ & $\begin{array}{c}\text { Do you feel } \\
\text { physically } \\
\text { healthy? }\end{array}$ & 100 & IN \\
\hline 2 & $\begin{array}{l}\text { Have you } \\
\text { lost a lot of } \\
\text { weight re- } \\
\text { cently without } \\
\text { wishing to } \\
\text { do so? ("a } \\
\text { lot"' is: 6kg } \\
\text { or more dur- } \\
\text { ing the last } \\
\text { six months or } \\
\text { 3kg or more } \\
\text { during the } \\
\text { last month) }\end{array}$ & $\begin{array}{c}\text { Você perdeu } \\
\text { muito peso re- } \\
\text { centemente sem } \\
\text { que desejasse? } \\
\text { (muito significa } \\
6 \text { kg ou mais } \\
\text { durante os últi- } \\
\text { mos seis meses } \\
\text { ou 3kg ou mais } \\
\text { durante o último } \\
\text { mês) }\end{array}$ & $\begin{array}{l}\text { Have you } \\
\text { unwittingly } \\
\text { lost a lot of } \\
\text { weight re- } \\
\text { cently? (6kg } \\
\text { or more in } \\
\text { the last six } \\
\text { months or } \\
3 \text { kg or more } \\
\text { in the last } \\
\text { month) }\end{array}$ & 80 & PA & $\begin{array}{l}\text { Você perdeu } \\
\text { muito peso re- } \\
\text { centemente sem } \\
\text { desejar que isso } \\
\text { acontecesse? } \\
\text { (muito é 6kg ou } \\
\text { mais nos últimos } \\
\text { seis meses ou } \\
\text { 3kg ou mais no } \\
\text { último mês) }\end{array}$ & $\begin{array}{c}\text { Have you } \\
\text { lost much } \\
\text { weight } \\
\text { recently } \\
\text { without } \\
\text { wanting this } \\
\text { to happen? } \\
\text { (Much is 6kg } \\
\text { or more in } \\
\text { the last six } \\
\text { months or } \\
3 \text { kg or more } \\
\text { in the last } \\
\text { month) }\end{array}$ & 90 & PA \\
\hline 3 & $\begin{array}{l}\text { Do you expe- } \\
\text { rience prob- } \\
\text { lems in your } \\
\text { daily life due } \\
\text { to: difficulty in } \\
\text { walking? }\end{array}$ & $\begin{array}{l}\text { Você tem tido } \\
\text { dificuldades } \\
\text { na sua vida } \\
\text { diária devido a: } \\
\text { dificuldade de } \\
\text { caminhar? }\end{array}$ & $\begin{array}{l}\text { Do you have } \\
\text { problems in } \\
\text { your daily } \\
\text { life due to: } \\
\text { difficulty walk- } \\
\text { ing? }\end{array}$ & 95 & IN & $\begin{array}{l}\text { Você tem pro- } \\
\text { blemas na sua } \\
\text { vida diária devi- } \\
\text { do a: dificuldade } \\
\text { de caminhar? }\end{array}$ & $\begin{array}{c}\text { Do you have } \\
\text { problems in } \\
\text { your daily } \\
\text { life due to: } \\
\text { difficulty } \\
\text { walking? }\end{array}$ & 95 & IN \\
\hline 4 & $\begin{array}{c}\text { Do you expe- } \\
\text { rience prob- } \\
\text { lems in your } \\
\text { daily life due } \\
\text { to: difficulty } \\
\text { in maintaining } \\
\text { your balance? }\end{array}$ & $\begin{array}{l}\text { Você tem tido } \\
\text { dificuldades na } \\
\text { sua vida diária } \\
\text { devido a: dificul- } \\
\text { dade em manter } \\
\text { seu equilíbrio? }\end{array}$ & $\begin{array}{l}\text { Do you have } \\
\text { problems in } \\
\text { your daily life } \\
\text { due to: diffi- } \\
\text { culty keeping } \\
\text { your balance? }\end{array}$ & 95 & IN & $\begin{array}{l}\text { Você tem pro- } \\
\text { blemas na sua } \\
\text { vida diária devi- } \\
\text { do a: dificuldade } \\
\text { de manter seu } \\
\text { equilíbrio? }\end{array}$ & $\begin{array}{c}\text { Do you have } \\
\text { problems in } \\
\text { your daily } \\
\text { life due to: } \\
\text { difficulty } \\
\text { keeping } \\
\text { your bal- } \\
\text { ance? }\end{array}$ & 95 & IN \\
\hline 5 & $\begin{array}{c}\text { Do you } \\
\text { experience } \\
\text { problems in } \\
\text { your daily life } \\
\text { due to: poor } \\
\text { hearing? }\end{array}$ & $\begin{array}{c}\text { Você tem tido } \\
\text { dificuldades } \\
\text { na sua vida } \\
\text { diária devido a: } \\
\text { deficiência de } \\
\text { audição? }\end{array}$ & $\begin{array}{l}\text { Do you have } \\
\text { problems in } \\
\text { your daily } \\
\text { life due to: } \\
\text { difficulty hear- } \\
\text { ing? }\end{array}$ & 95 & IN & $\begin{array}{l}\text { Você tem pro- } \\
\text { blemas na sua } \\
\text { vida diária devi- } \\
\text { do a: ouvir mal? }\end{array}$ & $\begin{array}{l}\text { Do you have } \\
\text { problems in } \\
\text { your daily } \\
\text { life due to: } \\
\text { hard of hear- } \\
\text { ing? }\end{array}$ & 95 & IN \\
\hline 6 & $\begin{array}{c}\text { Do you } \\
\text { experience } \\
\text { problems in } \\
\text { your daily life } \\
\text { due to: poor } \\
\text { vision? }\end{array}$ & $\begin{array}{l}\text { Você tem tido } \\
\text { dificuldades na } \\
\text { sua vida diária } \\
\text { devido a: defici- } \\
\text { ência de visão? }\end{array}$ & $\begin{array}{l}\text { Do you have } \\
\text { problems in } \\
\text { your daily life } \\
\text { due to: diffi- } \\
\text { culty seeing? }\end{array}$ & 90 & PA & $\begin{array}{c}\text { Você tem pro- } \\
\text { blemas na sua } \\
\text { vida diária devi- } \\
\text { do a: enxergar } \\
\text { mal? }\end{array}$ & $\begin{array}{c}\text { Do you have } \\
\text { problems in } \\
\text { your daily } \\
\text { life due to: } \\
\text { poor eye- } \\
\text { sight? }\end{array}$ & 90 & PA \\
\hline
\end{tabular}

(continua) 
Tabela 1 (continuação)

\begin{tabular}{|c|c|c|c|c|c|c|c|c|c|}
\hline Item & Original & 1ạ tradução & 1 a retradução & $\%$ * & $\begin{array}{l}\text { Avaliação do } \\
\text { significado } \\
\text { geral ** }\end{array}$ & 2a tradução & $\begin{array}{c}\text { 2a re- } \\
\text { tradução }\end{array}$ & $\%$ * & $\begin{array}{l}\text { Avaliação do } \\
\text { significado } \\
\text { geral ** }\end{array}$ \\
\hline 7 & $\begin{array}{c}\text { Do you } \\
\text { experience } \\
\text { problems in } \\
\text { your daily life } \\
\text { due to: lack } \\
\text { of strength in } \\
\text { your hands? }\end{array}$ & $\begin{array}{l}\text { Você tem tido } \\
\text { dificuldades na } \\
\text { sua vida diária } \\
\text { devido a: perda } \\
\text { de força nas } \\
\text { suas mãos? }\end{array}$ & $\begin{array}{l}\text { Do you have } \\
\text { problems in } \\
\text { your daily life } \\
\text { due to: weak- } \\
\text { er hands? }\end{array}$ & 85 & PA & $\begin{array}{l}\text { Você tem pro- } \\
\text { blemas na sua } \\
\text { vida diária devi- } \\
\text { do a: perda de } \\
\text { força nas mãos? }\end{array}$ & $\begin{array}{c}\text { Do you have } \\
\text { problems in } \\
\text { your daily } \\
\text { life due to: } \\
\text { loss of } \\
\text { strength in } \\
\text { your hands? }\end{array}$ & 90 & IN \\
\hline 8 & $\begin{array}{l}\text { Do you expe- } \\
\text { rience prob- } \\
\text { lems in your } \\
\text { daily life due } \\
\text { to: physical } \\
\text { tiredness? }\end{array}$ & $\begin{array}{l}\text { Você tem tido } \\
\text { dificuldades na } \\
\text { sua vida diária } \\
\text { devido a: cansa- } \\
\text { ço físico? }\end{array}$ & $\begin{array}{l}\text { Do you have } \\
\text { problems in } \\
\text { your daily life } \\
\text { due to: physi- } \\
\text { cal tiredness? }\end{array}$ & 95 & IN & $\begin{array}{l}\text { Você tem pro- } \\
\text { blemas na sua } \\
\text { vida diária de- } \\
\text { vido a: cansaço } \\
\text { físico? }\end{array}$ & $\begin{array}{c}\text { Do you have } \\
\text { problems in } \\
\text { your daily } \\
\text { life due to: } \\
\text { physical fa- } \\
\text { tigue? }\end{array}$ & 95 & IN \\
\hline 9 & $\begin{array}{l}\text { Do you have } \\
\text { problems } \\
\text { with your } \\
\text { memory? }\end{array}$ & $\begin{array}{c}\text { Você tem pro- } \\
\text { blemas de me- } \\
\text { mória? }\end{array}$ & $\begin{array}{c}\text { Do you have } \\
\text { memory } \\
\text { problems? }\end{array}$ & 95 & PA & $\begin{array}{c}\text { Você tem pro- } \\
\text { blemas de me- } \\
\text { mória? }\end{array}$ & $\begin{array}{c}\text { Do you have } \\
\text { memory } \\
\text { problems? }\end{array}$ & 95 & PA \\
\hline 10 & $\begin{array}{l}\text { Have you felt } \\
\text { down dur- } \\
\text { ing the last } \\
\text { month? }\end{array}$ & $\begin{array}{l}\text { Você se sentiu } \\
\text { deprimido du- } \\
\text { rante o último } \\
\text { mês? }\end{array}$ & $\begin{array}{l}\text { Have you felt } \\
\text { down during } \\
\text { the previous } \\
\text { month? }\end{array}$ & 95 & IN & $\begin{array}{c}\text { Você se sentiu } \\
\text { para baixo no } \\
\text { último mês? }\end{array}$ & $\begin{array}{c}\text { Did you feel } \\
\text { down last } \\
\text { month? }\end{array}$ & 100 & IN \\
\hline 11 & $\begin{array}{l}\text { Have you felt } \\
\text { nervous or } \\
\text { anxious dur- } \\
\text { ing the last } \\
\text { month? }\end{array}$ & $\begin{array}{l}\text { Você se sentiu } \\
\text { nervoso ou an- } \\
\text { sioso durante o } \\
\text { último mês? }\end{array}$ & $\begin{array}{l}\text { Have you } \\
\text { been anxious } \\
\text { or nervous } \\
\text { last month? }\end{array}$ & 100 & IN & $\begin{array}{l}\text { Você se sentiu } \\
\text { nervoso ou an- } \\
\text { sioso no último } \\
\text { mês? }\end{array}$ & $\begin{array}{l}\text { Did you feel } \\
\text { nervous or } \\
\text { anxious last } \\
\text { month? }\end{array}$ & 100 & IN \\
\hline 12 & $\begin{array}{c}\text { Are you able } \\
\text { to cope with } \\
\text { problems } \\
\text { well? }\end{array}$ & $\begin{array}{c}\text { Você consegue } \\
\text { lidar com os } \\
\text { seus problemas? }\end{array}$ & $\begin{array}{l}\text { Are you ca- } \\
\text { pable of deal- } \\
\text { ing well with } \\
\text { problems? }\end{array}$ & 90 & PA & $\begin{array}{c}\text { Você é capaz de } \\
\text { lidar bem com } \\
\text { os problemas? }\end{array}$ & $\begin{array}{c}\text { Are you able } \\
\text { to cope with } \\
\text { problems? }\end{array}$ & 100 & IN \\
\hline 13 & $\begin{array}{c}\text { Do you live } \\
\text { alone? }\end{array}$ & Você vive só? & $\begin{array}{c}\text { Do you live } \\
\text { alone? }\end{array}$ & 100 & IN & $\begin{array}{l}\text { Você vive } \\
\text { sozinho? }\end{array}$ & $\begin{array}{c}\text { Do you live } \\
\text { alone? }\end{array}$ & 100 & IN \\
\hline 14 & $\begin{array}{l}\text { Do you some- } \\
\text { times miss } \\
\text { having peo- } \\
\text { ple around } \\
\text { you? }\end{array}$ & $\begin{array}{l}\text { Você algumas } \\
\text { vezes sente falta } \\
\text { de ter pessoas } \\
\text { ao seu redor? }\end{array}$ & $\begin{array}{c}\text { Do you some- } \\
\text { times miss } \\
\text { having peo- } \\
\text { ple around } \\
\text { you? }\end{array}$ & 100 & IN & $\begin{array}{c}\text { Você algumas } \\
\text { vezes sente falta } \\
\text { de ter pessoas } \\
\text { ao seu redor? }\end{array}$ & $\begin{array}{l}\text { Do you } \\
\text { sometimes } \\
\text { miss hav- } \\
\text { ing people } \\
\text { around you? }\end{array}$ & 100 & IN \\
\hline 15 & $\begin{array}{l}\text { Do you re- } \\
\text { ceive enough } \\
\text { support from } \\
\text { other people? }\end{array}$ & $\begin{array}{c}\text { Você recebe } \\
\text { apoio suficiente } \\
\text { de outras pes- } \\
\text { soas? }\end{array}$ & $\begin{array}{c}\text { Do you get } \\
\text { enough sup- } \\
\text { port from } \\
\text { other people? }\end{array}$ & 95 & IN & $\begin{array}{c}\text { Você recebe } \\
\text { apoio suficiente } \\
\text { de outras pes- } \\
\text { soas? }\end{array}$ & $\begin{array}{l}\text { Do you } \\
\text { receive } \\
\text { enough sup- } \\
\text { port from } \\
\text { other peo- } \\
\text { ple? }\end{array}$ & 100 & IN \\
\hline
\end{tabular}

* Avaliação do significado referencial por meio do percentual de correspondência literal de cada par de perguntas, com pontuação entre 0 a $100 \%$;

** Segundo categorias: IN (inalterado); PA (pouco alterado); MA (muito alterado); CA (completamente alterado). 
Tilburg Frailty Indicator (TIF) adaptado para o Brasil (itens escolhidos, versão final e alterações).

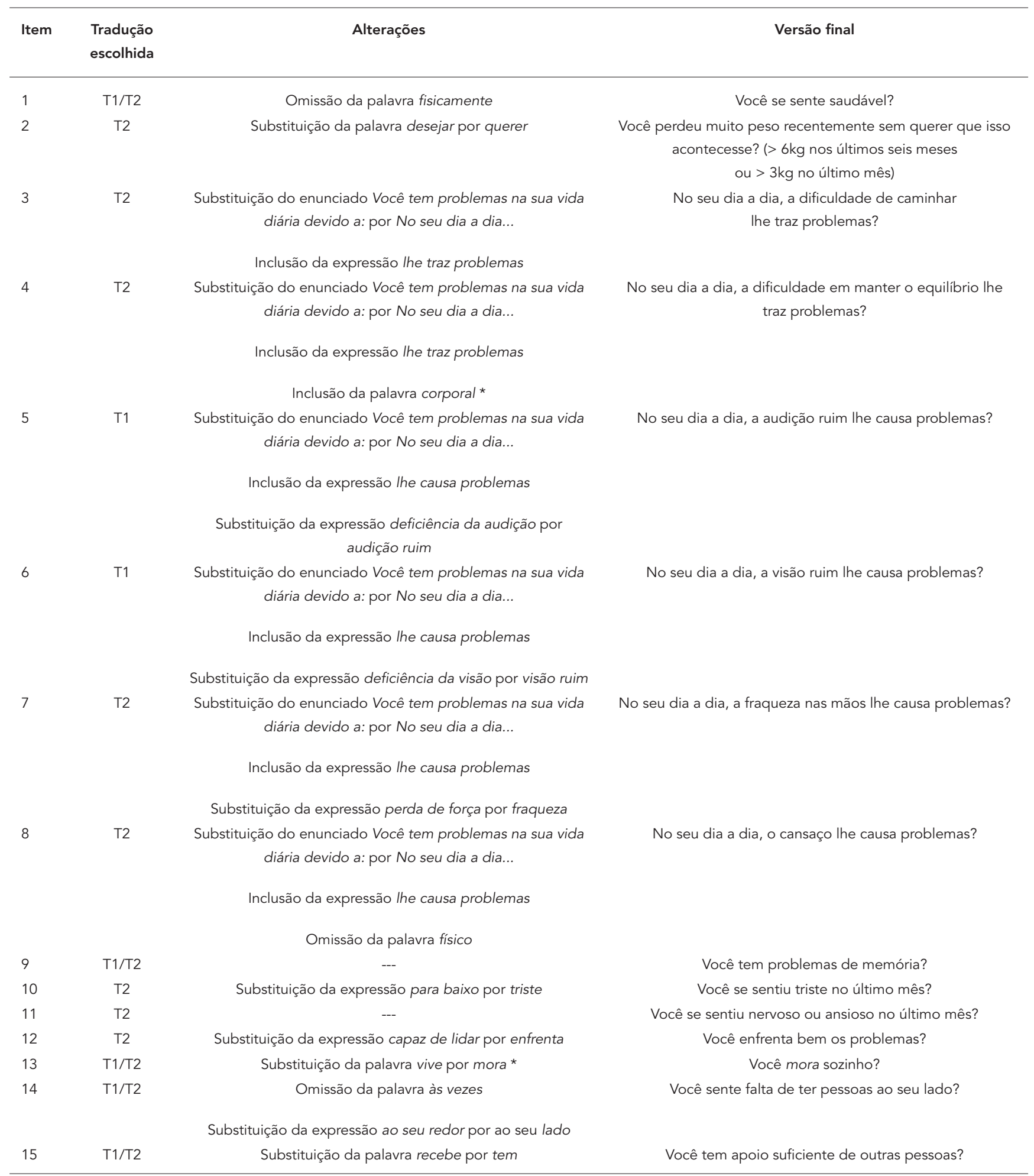

T1: tradução 1; T2: tradução 2.

* Modificado após pré-teste. 
"visão ruim", por considerar que a palavra "deficiência” poderia ser mal recebida.

No item 7, a expressão "perda de força" foi substituída por "fraqueza", para simplificação da questão. No item 8, omitiu-se a palavra "físico", porque a mesma já se encontrava implícita na questão.

Os itens 9 a 12 demandaram poucas discussões por serem de fácil compreensão em ambos os idiomas. Entretanto, foram realizadas pequenas alterações: no item 10, substituição da expressão "para baixo" por "triste”, com significado semelhante na língua portuguesa; no item 12, substituição da expressão “capaz de lidar” pela palavra "enfrenta", muito utilizada na linguagem popular nesse contexto.

Os três últimos itens sofreram somente adequações para que as sentenças fossem simplificadas. No item 14, omitiu-se a expressão "às vezes”, por representar uma das opções de resposta à questão. No item 15, substituiu-se "recebe" por "tem”, porque, na língua portuguesa, a primeira é um sinônimo de "ganhar", o que poderia gerar dúvidas de interpretação da palavra "apoio" como financeiro e não relacionado ao social.

A versão-síntese foi aplicada a 30 indivíduos idosos residentes na comunidade, sendo observadas boa aceitação e compreensão do instrumento.

Os resultados do pré-teste mostraram que 13 itens alcançaram $100 \%$ de entendimento. No item 4 , três dos entrevistados manifestaram dúvidas sobre o tipo de equilíbrio ao qual a questão fazia referência (mental ou físico); e, no item 13, três manifestaram dúvidas sobre o significado da palavra "vive".

Em reunião dos especialistas para a avaliação do pré-teste, essas questões foram alvo de discussão. Considerando que o problema colocado no item estava relacionado ao tipo de equilíbrio, optou-se por incluir a palavra "corporal". Quanto ao item 13, percebeu-se que a dificuldade de compreensão estava vinculada ao sentimento de solidão, e decidiu-se substituir "vive" por “mora”, já que se desejava aferir se o idoso residia sozinho.

\section{Discussão}

O constructo fragilidade adotado no Brasil é concebido de forma similar ao de outras culturas e inclui variáveis comumente consideradas em avaliações de saúde do idoso brasileiro 1,10.

O grupo de especialistas que realizou o presente estudo considerou que os domínios físico, social e psicológico do TFI e seus itens eram pertinentes para a análise da fragilidade em idosos brasileiros.

Observou-se alta equivalência entre as retraduções efetuadas para este estudo tanto em relação ao significado denotativo quanto ao conotativo. Embora as duas retraduções tenham apresentado boa qualidade, grande parte das questões escolhidas para compor a versão-síntese foi oriunda da tradução dois, que apresentava linguagem mais objetiva.

Como o público-alvo do instrumento é heterogêneo quanto à escolaridade, decidiu-se introduzir algumas modificações nas questões para simplificá-las gramaticalmente. Ainda, algumas alterações realizadas visaram à adequação do vocabulário para uma linguagem mais coloquial. A participação do pesquisador principal do grupo do TFI foi importante para que houvesse coerência entre a versão original e a adaptada.

O elevado percentual de entendimento e a boa aceitação dos itens do instrumento sugerem que o TFI adaptado possa ser uma ferramenta útil na avaliação dos idosos brasileiros. Entretanto, se faz necessária a continuidade desse processo com a avaliação das propriedades psicométricas apresentadas pelo instrumento traduzido para que seja assegurada a sua validade. A etapa de avaliação da equivalência de mensuração do instrumento já está sendo realizada e será oportunamente divulgada. 


\section{Resumo}

Este estudo tem como objetivo realizar a adaptação para a população brasileira do Tilburg Frailty Indicator (TFI), instrumento utilizado para a avaliação da fragilidade em idosos. Foram realizadas análises das equivalências conceitual, de itens e semântica, além do pré-teste da versão-síntese do instrumento. Na avaliação da equivalência conceitual, pôde-se constatar que o constructo fragilidade adotado no Brasil tem a mesma concepção das demais culturas onde essa condição tem sido investigada. Verificou-se que todos os itens incluídos no instrumento original apresentavam similaridade de conteúdo nas duas culturas. Identificou-se, também, alta equivalência semântica tanto na análise do significado referencial quanto do significado geral dos itens. No pré-teste, foram observados elevado percentual de entendimento e boa aceitação dos itens do instrumento por parte dos idosos. Os resultados sugerem que a versão do TFI para a cultura brasileira pode representar uma ferramenta útil na avaliação da saúde dos idosos do país.

Avaliação; Fragilidade; Idoso

\section{Referências}

1. van Kan G, Rolland Y, Bergman H, Morley JE, Kritchevsky SB, Vellas B, et al. The IANA task force on frailty assessment of older people in clinical practice. J Nutr Health Aging 2008; 12:29-37.

2. Fried LP, Tangen CM, Walston J, Newman AB, Hirsch C, Gottdiener J, et al. Frailty in older adults: evidence for a phenotype. J Gerontol Med Sci 2001; 56:M146-56.

3. Ensrud KE, Ewing SK, Taylor BC, Fink HA, Cawthon PM, Stone KL, et al. Comparison of 2 frailty indexes for prediction of falls, disability, fractures, and death in older women. Arch Intern Med 2008; 168:382-9.

4. Rothman MD, Leo-Summers L, Gill TM. Prognostic significance of potential frailty criteria. J Am Geriatr Soc 2008; 56:2211-6.

5. Gobbens RJJ, Luijkx KG, Wijnen-Sponselee MT, Schols JMGA. In search of an integral conceptual definition of frailty: opinions of experts. J Am Med Dir Assoc 2010; 11:338-43.

\section{Colaboradores}

L. M. Santiago participou do desenvolvimento teórico, revisão bibliográfica, organização do trabalho de campo, discussão e análise dos resultados e elaboração da redação do artigo. L. L. Luz participou do desenvolvimento teórico, discussão dos dados e elaboração da redação do artigo. I. E. Mattos participou do desenvolvimento teórico, revisão bibliográfica, discussão e análise dos dados e redação do artigo. R. J. J. Gobbens participou do desenvolvimento teórico, escolha metodológica e revisão do artigo.
6. Strawbridge WJ, Shema SJ, Balfour JL, Higby HR, Kaplan GA. Antecedents of frailty over three decades in an older cohort. J Gerontol A Biol Sci Med Sci 1998; 53B:S9-16.

7. Gobbens RJJ, van Assen MALM, Luijkx KG, WijnenSponselee MT, Schols JMGA. Determinants of frailty. J Am Med Dir Assoc 2010; 11:356-64.

8. Herdman M, Fox-Rushbay J, Badia X. A model of equivalence in the cultural adaptation of HRQoL instruments: the universalist approach. Qual Life Res 1998; 7:323-35.

9. Reichenheim ME, Moraes CL. Operacionalização de adaptação transcultural de instrumentos de aferição usados em epidemiologia. Rev Saúde Pública 2007; 41:665-73.

10. Teixeira INDO. Percepções de profissionais de saúde sobre duas definições de fragilidade no idoso. Ciênc Saúde Coletiva 2008; 13:1181-8.

Recebido em 07/Abr/2012

Versão final reapresentada em 13/Mai/2012

Aprovado em 25/Jun/2012 